\title{
Mortalidade por síndrome de aspiração meconial em recém-nascidos no estado do Pará, Região Norte do Brasil
}

\author{
Mortality by meconial aspiration syndrome in newborn in the state of Pará, Northern Brazil
}

Mortalidad por síndrome de aspiración meconial en el recién nacido en el estado de Pará, Norte de Brasil

Anildo de Sousa Carvalho ${ }^{1}$, Jefferson Maia Franco ${ }^{1}$, Maria Celina Borges Maciel ${ }^{1}$, Samuel Filipe Lopes Alves ${ }^{2}$, Pedro Rafael Rocha Stermer ${ }^{2}$, Luiz Euclides Coelho de Souza Filho², Amanda Gomes Chermont ${ }^{3}$, Aurimery Gomes Chermont ${ }^{1}$, Katiane da Costa Cunha ${ }^{2 *}$.

\section{RESUMO}

Objetivo: Identificar a mortalidade por Síndrome de Aspiração Meconial (SAM) em recém-nascidos no estado do Pará, no período de 1996 a 2016. Métodos: Trata-se de estudo analítico, quantitativo e retrospectivo, que utilizou como banco de dados secundários o Sistema de Informações sobre Mortalidade (SIM), disponível ao acesso público por meio do Tabulador do Windows (TABNET), uma ferramenta do DATASUS. A tabulação dos dados foi feita com o auxílio do software Tabwin, o Excel® foi utilizado para construção de gráficos e tabelas, e o Quantum GIS para o mapeamento. Resultados: No período pesquisado ocorreram 26.812 óbitos por SAM em RN no Brasil, sendo que o Pará é o 6o estado brasileiro com maior número de óbitos por SAM em RN ( $n=1.507)$ representando $6 \%$ do total nacional e $46 \%$ dos óbitos por SAM na Região Norte e em números relativos é o $10^{\circ}$ com o maior índice (TM=0,53/1000). Conclusão: O Pará apresenta índices significativos de óbitos por SAM, contudo podem estar sendo subestimados devido a possível subnotificação.

Palavras-chave: Síndrome de Aspiração Meconial, Recém-nascido, Mortalidade.

\begin{abstract}
Objective: To identify the mortality by Meconium Aspiration Syndrome (MAS) in newborns in the State of Pará, from 1996 to 2016. Methods: This is an analytical, quantitative and retrospective study that used as a secondary database the Mortality Information System (SIM), available to the public through Tabulador do Windows (TABNET), a DATASUS tool. Data were tabulated using the software Tabwin, Excel ${ }^{\circledR}$ was used to construct graphs and tables, and Quantum GIS for mapping. Results: In the surveyed period, there were 26,812 deaths from MAS in newborns in Brazil, and Pará is the 6th Brazilian state with the highest number of deaths from MAS in newborns $(n=1,507)$ representing $6 \%$ of the national total and $46 \%$ of deaths from SAM in the state. North region. And in relative numbers is the 10th with the highest index (MR=0.53/1000). Conclusion: Pará has significant SAM mortality rates but may be underestimated due to possible underreporting.
\end{abstract}

Keywords: Meconium Aspiration Syndrome, Newborn, Mortality.

1 Universidade Federal do Pará (UFPA), Belém-PA.

2Universidade do Estado do Pará (UEPA), Belém-PA. *E-mail: katianefisio@yahoo.com.br.

${ }^{3}$ Maternidade Saúde da Criança (MSC), Belém-PA. 


\section{RESUMEN}

Objetivo: Identificar la mortalidad por Síndrome de Aspiración Mecónica (SAM) en recién nacidos en el estado de Pará, de 1996 a 2016. Métodos: Este es un estudio analítico, cuantitativo y retrospectivo que utilizó como base de datos secundaria el Sistema de información de mortalidad (SIM), disponible al público a través de Tabulador do Windows (TABNET), una herramienta DATASUS. Los datos se tabularon usando el software Tabwin, Excel® se utilizó para construir gráficos y tablas, y Quantum GIS para el mapeo. Resultados: En el período encuestado hubo 26.812 muertes por SAM en recién nacidos en Brasil, y Pará es el sexto estado brasileño con el mayor número de muertes por SAM en recién nacidos $(n=1,507)$ que representa el $6 \%$ del total nacional y el $46 \%$ de las muertes por recién nacidos. SAM en la Región Norte y en números relativos es el décimo con el índice más alto $(T M=0.53 / 1000)$. Conclusión: Pará tiene tasas de mortalidad significativas debido a SAM, pero puede subestimarse debido a un posible subregistro.

Palabras clave: Síndrome de aspiración de meconio, Recién nacido, Mortalidad.

\section{INTRODUÇÃO}

A Síndrome de Aspiração de Mecônio (SAM) é uma causa frequente de morbi-mortalidade em neonatos, caracteriza-se por uma vasta amplitude do grau de insuficiência respiratória e é causada pela inalação de mecônio para o interior dos pulmões do feto ou recém-nascido (RN) (CHETTRI S, et al., 2016; HAHN S. et al., 2013; LINDENSKOV PH, et al., 2015).

O mecônio é uma matriz de agentes excretado pelo neonato, em situações fisiológicas normais, cerca de 48 horas depois do nascimento de bebês atermos. Trata-se de um material viscoso, inodor, de coloração verde-escura e composição variada, podendo conter fluido amniótico deglutido, produtos de degradação celular, secreções intestinais e sangue fetal ou materno ingeridos (LISOWSKA-MYJAK B, et al., 2018).

O mecônio raramente é encontrado no líquido amniótico antes de 34 semanas de gestação, sendo assim, a SAM é frequentemente uma patologia observada em neonatos a termo e predominantemente em pós-termos. O líquido amniótico meconial (LAM) está presente em $8-20 \%$ de todos os partos, aumentando para 23-52\% após 42 semanas de gestação (SWARNAM K, et al., 2012). Um neonato tem SAM se a tríade, líquido meconial, desconforto respiratório e características radiológicas típicas, são observados (ANAND V, et al., 2018; CHETTRI S, et al., 2016; SWARNAM K, et al., 2012).

Swarnam K, et al. (2012) afirmaram que a fisiopatologia da SAM é multifatorial e inclui obstrução aguda das vias aéreas, disfunção ou inativação do surfactante, pneumonite química com liberação de mediadores vasoconstritores e inflamatórios e hipertensão pulmonar persistente do recém-nascido (HPPN). A SAM pode ser fatal, muitas vezes complicado por insuficiência respiratória, extravasamento de ar dos pulmões e HPPN. Abordagens para a prevenção da SAM mudaram ao longo do tempo com a colaboração de obstetras e pediatras, formando as bases para o cuidado. O uso de surfactante e óxido nítrico inalatório (NOi) levou a diminuição da mortalidade e à necessidade de uso de oxigenação por membrana extracorpórea no manejo da SAM.

Quanto à classificação da SAM, Cleary GM e Wiswell TE(1998) propuseram três critérios para definição: SAM leve é uma doença que requer uma fração inspiratória de oxigênio ( $\mathrm{FiO} 2$ ) menor que $40 \%$ por menos de 48 horas; SAM moderada é uma doença que requer uma $\mathrm{FiO} 2$ maior que $40 \%$, sem extravasamento de ar, por mais de 48 horas; e SAM grave é uma doença que requer ventilação assistida por mais de 48 horas e é frequentemente associada a hipertensão pulmonar persistente do recém-nascido (HPPRN) (SWARNAM $\mathrm{K}$, et al., 2012).

Já em relação a gravidade, a SAM é uma importante causa de mortalidade e morbidade neonatal (BATRA P, et al., 2018). Cerca de $5 \%$ dos neonatos nascidos com líquido amniótico mecônio desenvolvem SAM (NARAYANAN A,et al., 2019) e, aproximadamente $26 \%$ desses pacientes vem a óbito nos primeiros dias de vida (LOUIS D, et al., 2014; NARAYANAN A, et al., 2019). 
Visto a sua importância clínica, é necessário mecanismos de intervenção para combater esta síndrome. Assim, a coordenação de cuidados entre a equipe obstétrica e neonatal é importante para reduzir a incidência de SAM e para identificar e fornecer tratamentos emergentes naqueles pacientes que desenvolveram SAM, no intuito de reduzir a morbidade e mortalidade (NARAYANAN A, et al., 2019; PRATS JAG, 2018).

Neste contexto, o objetivo da presente pesquisa foi verificar o índice de mortalidade por SAM em recémnascidos no Pará, um estado situado na região Norte do Brasil, cuja extensão territorial corresponde a $1.247 .689,515 \mathrm{~km}^{2}$, representando $16,66 \%$ do território brasileiro e $26 \%$ da Amazônia, no período de 1996 a 2016 (IBGE, 2019).

\section{MÉTODOS}

Trata-se de estudo analítico, quantitativo e retrospectivo, que utilizou como banco de dados secundários o Sistema de informações sobre Mortalidade (SIM), disponível ao acesso público por meio do TABNET, uma ferramenta do Departamento de Informática do Sistema Único de Saúde (DATASUS), que possui a função de coleta, organização e disponibilização dos dados em saúde. Por utilizar um banco de dados secundário de acesso público, não necessitou de avaliação pelo Conselho de Ética e da Comissão Nacional de Ética em Pesquisa CEP/CONEP, de acordo com a resolução de ํㅡㄴ 510, de 07 de abril de 2016.

A busca foi realizada no mês de março de 2019 e limitou-se a investigar os óbitos no período de 1996 a 2016. A população do estudo foi constituída por recém-nascidos que evoluíram a óbito por SAM, no período de 1996 a 2016, no estado paraense, considerando-se suas regiões de saúde e os municípios com mais de 100 mil habitantes.

Além de comparar os dados paraenses com aqueles de outros estados brasileiros. Vale ressaltar que o estado do Pará é dividido em 13 Regiões de saúde. As Regiões de saúde são um conjunto de municípios limítrofes que compartilham características culturais, econômicas ou sociais, agrupados a fim de promover a integração das estratégias de ação em saúde (BRASIL, 2011).

Para a coleta de dados acessou-se o site do TABNET, na página de Estatísticas Vitais DATASUS (1996 a 2016), escolhendo-se a opção "óbitos infantis", em seguida "Brasil por região e Unidade de Federação". Na linha fixou-se: Região e Região de Saúde (CIR), na coluna: faixa etária (menos de 1 hora; 1 a 11 horas; 12 a 23 horas; menos de 1 dia, número ignorado de horas; 1 dia; 2 dias; 3 a 6 dias; 7 dias ou mais) e no conteúdo: óbitos por ocorrência, período de 1996 a 2016. Posteriormente, a busca foi filtrada em categoria: Classificação Internacional de Doenças (CID)10 - P24.0 Aspiração neonatal de mecônio.

Quanto a análise dos dados, foi executada a tabulação por meio do software Tabwin, desenvolvido pelo Departamento de Informática do Sistema único de Saúde, a fim de facilitar a utilização dos dados disponíveis no TABNET. Foram utilizados cálculos de estatísticas simples, como o de frequência relativa e absoluta.

Além do cálculo de Taxa de Mortalidade (TM), no qual dividiu-se a frequência absoluta dos óbitos por SAM no período analisado (obtida no SIM) pela quantidade de nascidos vivos no mesmo período para os estados brasileiros e para as regiões de saúde e municípios paraenses analisados (retirada no SINASC). A tabulação dos dados foi realizada no programa Excel® e apresentada em forma de gráficos e tabelas, enquanto o mapa foi criado e editado no programa QGIS®.

\section{RESULTADOS}

No período de 1996 a 2016 foram registrados no Brasil 26.812 óbitos por Síndrome de Aspiração Meconial em neonatos, com uma média de 1235 óbitos por anos. A curva em relação ao tempo aponta uma tendência de queda da mortalidade por esta causa. 
O pico de maior incidência ocorreu em 1997 ( $n=1806)$ e a menor incidência ocorreu em 2016 ( $n=946)$, ou seja, uma queda de 47,6\% em 19 anos. Das regiões federativas brasileiras, a região Sudeste apresentou a maior quantidade absoluta de óbitos por SAM, com um total de $9381(35 \%)$ das mortes apontadas neste período.

Contudo, nessa região houve uma considerável diminuição da mortalidade por SAM nos primeiros sete anos do período analisado, fato que resultou na liderança da região Nordeste em praticamente todos os anos a partir de 2002. Em contrapartida, a região centro-oeste apresentou o menor número de óbitos, com 2347 casos (8,75\%). O gráfico abaixo apresenta a curva temporal dos registros de óbitos durante o período analisado, considerando as regiões federativas do Brasil (Gráfico 1).

Gráfico 1 - Óbitos por Síndrome de Aspiração Meconial em Recém-nascido por região brasileira,1996 a 2016. $(N=26812)$

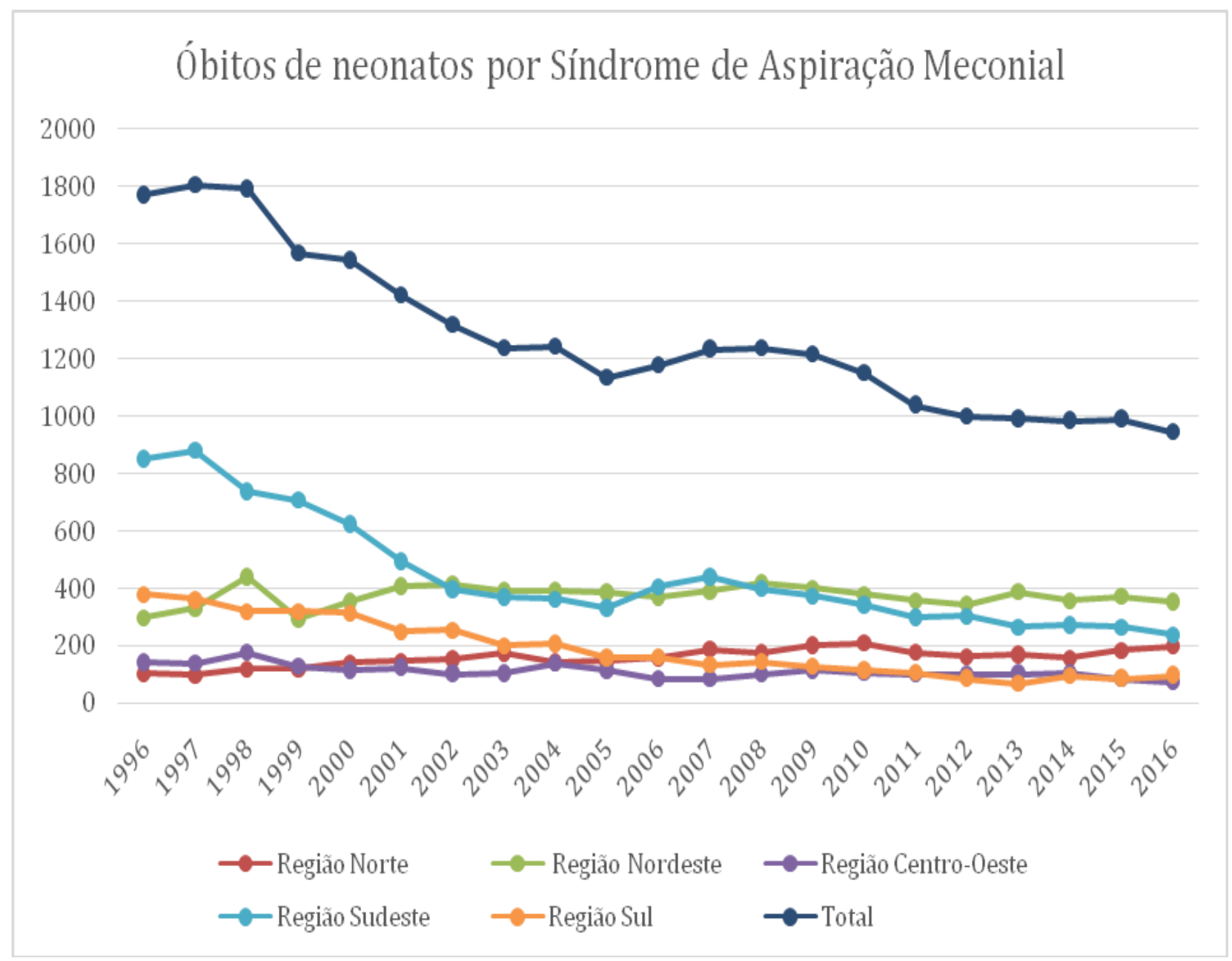

Fonte: Sistema de Nascidos Vivos - DATASUS, 2019.

Não obstante, a tabela abaixo descreve as taxas de mortalidade e as frequências absoluta e relativa de óbitos por SAM entre os estados brasileiros. Em ordem decrescente, o estado de São Paulo apresenta a maior frequência com $4399(16,4 \%)$ casos, seguido de Minas Gerais com 2571 (9,6\%), Paraná, que apresentou 1898 (7,1\%) casos, Rio de Janeiro com 1937 (7,2\%), Bahia, com 1724 (6,4\%) e Pará com 1507 $(5,6 \%)$ casos.Quanto a taxa de mortalidade, observa-se que os estados de Sergipe $(0,76)$, Rondônia $(0,70)$ e Tocantins $(0,68)$ apresentam os maiores indicadores (Tabela 1). 
Tabela 1 - Óbitos por Síndrome de Aspiração Meconial (SAM) em Recém-nascidos nos estados brasileiros, 1996 a 2016. $(\mathrm{N}=26812)$

\begin{tabular}{|c|c|c|c|c|}
\hline \multirow{2}{*}{$\begin{array}{l}\text { Estados brasileiros (em ordem } \\
\text { alfabética) }\end{array}$} & \multicolumn{2}{|c|}{ Óbitos } & \multirow{2}{*}{$\begin{array}{l}\text { População de } \\
\text { Nascidos vivos }\end{array}$} & \multirow{2}{*}{$\begin{array}{l}\text { TM por } 1.000 \\
\text { nascidos vivos }\end{array}$} \\
\hline & $\mathbf{N}$ & $\%$ & & \\
\hline Acre & 125 & 0,5 & 345630 & 0,36 \\
\hline Alagoas & 681 & 2,5 & 1211005 & 0,56 \\
\hline Amapá & 185 & 0,7 & 319255 & 0,58 \\
\hline Amazonas & 599 & 2,2 & 1487483 & 0,40 \\
\hline Bahia & 1724 & 6,4 & 4655139 & 0,37 \\
\hline Ceará & 953 & 3,6 & 2818998 & 0,34 \\
\hline Distrito Federal & 300 & 1,1 & 1188503 & 0,25 \\
\hline Espírito Santo & 474 & 1,8 & 1142339 & 0,41 \\
\hline Goiás & 1024 & 3,8 & 1739681 & 0,59 \\
\hline Maranhão & 1194 & 4,5 & 2300037 & 0,52 \\
\hline Mato Grosso & 553 & 2,1 & 1059434 & 0,52 \\
\hline Mato Grosso do Sul & 470 & 1,8 & 864969 & 0,54 \\
\hline Minas Gerais & 2571 & 9,6 & 5543368 & 0,46 \\
\hline Pará & 1507 & 5,6 & 2841669 & 0,53 \\
\hline Paraíba & 539 & 2,0 & 1209995 & 0,45 \\
\hline Paraná & 1898 & 7,1 & 3442122 & 0,55 \\
\hline Pernambuco & 1359 & 5,1 & 3135036 & 0,43 \\
\hline Piauí & 425 & 1,6 & 1108683 & 0,38 \\
\hline Rio de Janeiro & 1937 & 7,2 & 4930179 & 0,39 \\
\hline Rio Grande do Norte & 324 & 1,2 & 1083360 & 0,30 \\
\hline Rio Grande do Sul & 1144 & 4,3 & 3196221 & 0,36 \\
\hline Rondônia & 411 & 1,5 & 589893 & 0,70 \\
\hline Roraima & 110 & 0,4 & 204859 & 0,54 \\
\hline Santa Catarina & 937 & 3,5 & 1884379 & 0,50 \\
\hline São Paulo & 4399 & 16,4 & 13314110 & 0,33 \\
\hline Sergipe & 608 & 2,3 & 801333 & 0,76 \\
\hline Tocantins & 361 & 1,3 & 532639 & 0,68 \\
\hline Total & 26812 & 100,0 & 62950319 & 0,43 \\
\hline
\end{tabular}

Legenda: $\mathrm{N}$ - Frequência absoluta, TM - taxa de mortalidade.

Fonte: Sistema de Nascidos Vivos - DATASUS, 2019. 
Analisando-se especificamente a posição do estado do Pará em número de óbitos por SAM em recémnascidos, observa-se que embora ocupe a sexta posição dentre os estados brasileiros, ele garantiu a marca de primeiro lugar dentre aqueles da Região Norte do qual faz parte. Desse, modo a figura 1 expõe o estado do Pará é dividido em 13 regiões de saúde, enumeradas de 1 a 13 e localizadas pelo mapa abaixo. Nele, descreve-se a taxa de mortalidade entre as regiões de saúde paraenses, e demonstra que os índices variaram entre 0,2 e 1,1 no período estudado. Xingu e Tapajós foram as regiões de saúde que obtiveram as maiores taxas de mortalidade por SAM, 1,1 e 0,82 respectivamente. As regiões de saúde que obtiveram as menores taxas de mortalidade foram a Metropolitana I, Metropolitana III e Rio Caetés, com 0,38, 0,38 e 0,30 , respectivamente (Figura 1).

Figura 1 - Taxa de mortalidade por SAM por 10000 nascidos vivos nas Regiões de Saúde do estado do Pará de 1996 a $2016(\mathrm{~N}=1507)$.

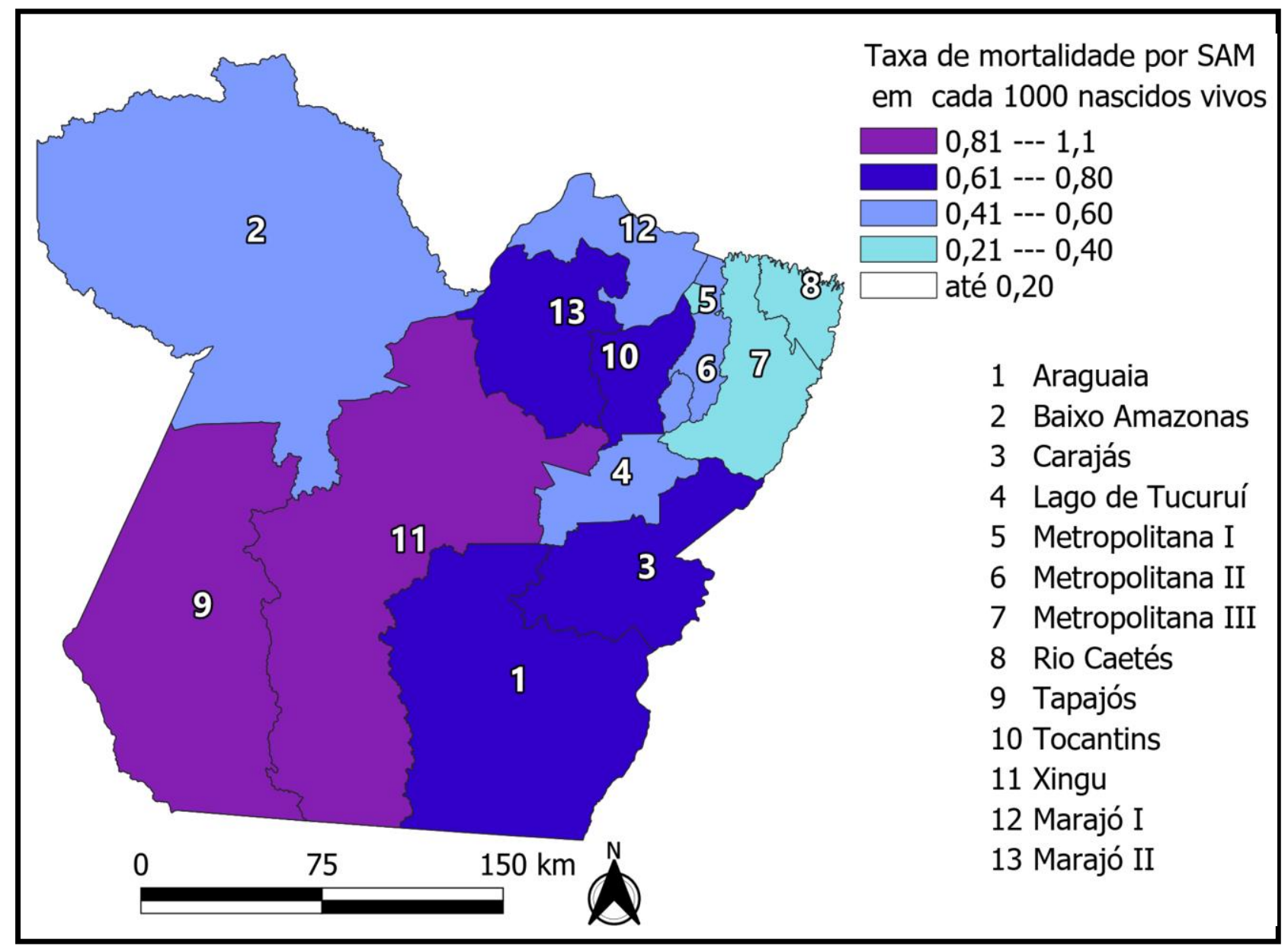

Fonte: Sistema de Nascidos Vivos- DATASUS, 2019.

O estado do Pará possui 144 municípios, dos quais 93 (65\%) registraram óbitos por SAM em RN no período de 1996 a 2016. O município com maior número de óbitos no período foi Belém ( $n=336 ; 22 \%$ ), com 1.393.399 habitantes desde o último Censo em 2010. Os 19 municípios com mais de 100.000 habitantes no estado do Pará, incluindo Belém, totalizaram 1.169 óbitos por SAM em RN no período de 1996 a 2016, outros 74 municípios, com menos de 100.000 habitantes, totalizaram 338 óbitos por SAM em RN no período estudado, e os demais municípios ( $n=51$ ) não apresentaram casos de óbitos por SAM em RN entre 1996 a 2016. A tabela 2 descreve os 19 municípios com mais de 100.000 habitantes que respondem por $78 \%$ dos óbitos por SAM em RN no período de 1996 a 2016 no estado do Pará (Tabela 2). 
Tabela 2 - Óbitos por SAM em RN nos 19 municípios com população superior a 100.000 habitantes no estado do Pará, 1996 a 2016. ( $N=1169)$

\begin{tabular}{|c|c|c|c|c|}
\hline \multirow{2}{*}{ Municípios (ordem alfabética) } & \multicolumn{2}{|c|}{ Óbitos } & \multirow{2}{*}{$\begin{array}{l}\text { População de } \\
\text { nascidos vivos }\end{array}$} & \multirow{2}{*}{$\begin{array}{l}\text { TM por } 1.000 \\
\text { nascidos } \\
\text { vivos }\end{array}$} \\
\hline & $\mathbf{N}$ & $\%$ & & \\
\hline Abaetetuba & 22 & $1,9 \%$ & 54109 & 0,41 \\
\hline Altamira & 66 & $5,6 \%$ & 61546 & 1,07 \\
\hline Ananindeua & 50 & $4,3 \%$ & 130778 & 0,38 \\
\hline Barcarena & 6 & $0,5 \%$ & 32992 & 0,18 \\
\hline Belém & 336 & $28,7 \%$ & 662847 & 0,51 \\
\hline Bragança & 38 & $3,3 \%$ & 68947 & 0,55 \\
\hline Breves & 59 & $5,0 \%$ & 45615 & 1,29 \\
\hline Cametá & 91 & $7,8 \%$ & 53081 & 1,71 \\
\hline Castanhal & 37 & $3,2 \%$ & 106643 & 0,35 \\
\hline Itaituba & 49 & $4,2 \%$ & 52831 & 0,93 \\
\hline Marabá & 98 & $8,4 \%$ & 102426 & 0,96 \\
\hline Marituba & 25 & $2,1 \%$ & 38568 & 0,65 \\
\hline Paragominas & 12 & $1,0 \%$ & 40671 & 0,30 \\
\hline Parauapebas & 60 & $5,1 \%$ & 74834 & 0,80 \\
\hline Redenção & 56 & $4,8 \%$ & 42437 & 1,32 \\
\hline Santarém & 99 & $8,5 \%$ & 141967 & 0,70 \\
\hline São Félix do Xingu & 12 & $1,0 \%$ & 15017 & 0,80 \\
\hline Tailândia & 13 & $1,1 \%$ & 26599 & 0,49 \\
\hline Tucuruí & 40 & $3,4 \%$ & 51670 & 0,77 \\
\hline Total & 1169 & $100,0 \%$ & 1803578 & 0,65 \\
\hline
\end{tabular}

Legenda: N- Frequência absoluta, TM- taxa de mortalidade

Fonte: Sistema de Nascidos Vivos - DATASUS, 2019. 


\section{DISCUSSÃO}

Os achados da presente pesquisa apontaram 26.812 óbitos por Síndrome de Aspiração Meconial no Brasil entre 1996 e 2016, com uma média de 1235 óbitos por ano. A região Sudeste destacou-se dentre as demais até o ano 2002, quando a região Nordeste passou a liderar os registros de óbitos por SAM. Na análise por estados, São Paulo apresentou a maior frequência de casos, enquanto Sergipe, Rondônia e Tocantins apresentam as maiores taxas de mortalidade. Sobre o estado do Pará, ele foi líder em casos de óbitos por SAM dentro da Região Norte, sendo o sexto dentre os estados brasileiros.

Sobre as 13 Regiões de Saúde paraenses, Xingu e Tapajós foram aquelas que obtiveram as maiores taxas de mortalidade por Síndrome de Aspiração Meconial, enquanto as regiões Metropolitana I, Metropolitana III e Rio Caetés registraram as menores taxas de mortalidade. Além disso dos 144 municípios do estado do Pará, 93 (65\%) registraram óbitos por SAM, com destaque para Belém e mais 18 cidades com mais de 100.000 habitantes que corresponderam a $78 \%$ dos óbitos por SAM em recém nascidos no período de 1996 a 2016.

A Síndrome de Aspiração Meconial (SAM) é uma das enfermidades mais importantes que ocorrem em recém-nascidos, principalmente pós-termos. Além disso, ela apresenta um nível alto de mortalidade, cerca de 26\% (NARAYANAN A, et al., 2019; PRATS JAG, 2018). Visto isso, é importante a produção cientifica acerca do tema, a fim de criar um arcabouço científico que possa ter a função de instrumento para auxiliar no enfrentamento desta patologia. Deste modo, fazendo uma análise voltada, inicialmente, a todo o território brasileiro, os índices de morte por SAM foram analisados no estudo de Chettri S, et al. (2016), que relatou uma taxa de $20 \%$ de óbitos por esta causa em recém-nascidos, nos países em desenvolvimento.

Contudo, os resultados desta pesquisa demonstraram que a SAM foi responsável pelos óbitos de 26.812 $\mathrm{RN}$ em todo o Brasil, ou seja, aproximadamente $2 \%$ de todas as causas de morte em crianças na faixa etária. Esta diferença está relacionada, possivelmente, com a subnotificação destes casos no Brasil. Isto porque a subnotificação pode mascarar os resultados de uma pesquisa, apresentando um valor positivo irreal, pois diminui a quantidade de óbitos em geral ou afeta o registro das causas dessa morte. Visto isso, o artigo de França EB, et al. (2017) apresenta também a subnotificação como uma das causas das diferenças regionais de óbito por causa de morte, além de corroborar com o fato de que o avanço nas técnicas de notificação tem proporcionado uma melhora contínua na coleta dos índices durante o período estudado pelo artigo.

Outro ponto que deve também ser exposto sobre essa possibilidade é o fato de grande parte das notificações acerca da causa de morte de neonatos ser registrada como "causas mal definidas" ou "demais causas", que totalizam cerca de $23 \%$ das notificações de óbitos na faixa etária de 0 a 27 dias de vida (DATASUS). Parte destas notificações podem ser casos de dúvidas acerca do diagnóstico da morte ou incapacidade de identificar a causa propriamente dita.

A Região Norte representou 12\% (3.208) de todas as mortes por SAM em RN no período de 1996 a 2016, ocupando o $4^{\circ}$ lugar no ranking nacional de mortalidade por SAM, este fato apresenta uma posição relativamente baixa em relação ao número absoluto de óbitos pela causa. Isso é constatado provavelmente devido a menor densidade de nascidos vivos, representando cerca de $10 \%$ dos nascidos de todo o país, sendo que o estado de São Paulo sozinho possui o dobro registros de nascidos vivos, totalizando $20 \%$ do total. Por esse motivo o cálculo da Taxa de Mortalidade (TM) se mostra útil para descrever mais fidedignamente a carga dessa patologia na saúde da população neonatal.

A média da TM entre os estados da Região Norte é a mais alta das cinco regiões federativas que compõem o território brasileiro, correspondendo a aproximadamente 0,54 óbitos por 1000 nascidos vivos. A efeito de comparação, a segunda região que possui maior média de TM entre seus estados é a Região Centro-Oeste, com 0.475 óbitos por 1000 nascidos vivos. A Região Sudeste apresenta a menor média de TM, com aproximadamente 0,39 óbitos por 1000 nascidos vivos. Quanto a análise dos estados que compõe a Região Norte separadamente, percebe-se que o Pará possui o maior número de óbitos por SAM nessa região, apresentando 46\% (1507) na região e 5,6\% dos óbitos por SAM no Brasil. Amazonas é o estado que 
possui o segundo maior número absoluto de óbitos por essa causa, somando 12\% (599) desses óbitos na Região Norte.

Entretanto, o estado do Pará também possui a maior população de nascidos vivos na Região Norte, contabilizando aproximadamente $44 \%$ dessa população na região. A TM do estado do Pará é de 0,53 mortes a cada 1000 nascidos vivos. Isto o coloca na décima posição na lista das maiores TM de todo o Brasil. Na Região Norte, o estado do Pará ocupa a quinta posição dentre os estados que possuem as maiores TM que é liderada pelo estado de Rondônia $(0,70)$, Tocantins $(0,68)$ e Amapá $(0,58)$.

Contudo, os dados do DATASUS não compreendem a totalidade de caso de SAM, apenas os casos que resultaram em óbito. Dessa forma, para se discutir sobre os índices de mortalidade da síndrome, é possível comparar estes dados com os achados da literatura. Swarnam K, et al. (2012) afirmaram que o líquido amniótico mecônio (LAM) está presente em 8 e $20 \%$ de todos os partos, ou seja, em média 14\% de todos os partos apresentam LAM; e que 2-9\% das crianças nascidas em LAM desenvolvem a SAM, portanto, uma média de $5,5 \%$. Com base nesses valores percentuais, considerando-se o percentual de $14 \%$ dos nascidos vivos no estado do Pará apresentaria LAM, 400.689 RN nasceriam com essas condições, sendo que 5,5\% deles (22.038) desenvolveriam SAM. Ao considerar a estimativa de 22.038 casos diagnosticados com SAM no Pará entre 1996-2016, o índice estimado de mortalidade por SAM seria de 6,84\%. Este número novamente aponta uma possível subnotificação dos casos.

No tratamento da SAM em RN, as terapias de suporte respiratório como suplementação de oxigênio, ventilação mecânica e fluídos intravenosos são consideradas o padrão ouro. A disponibilidade de surfactante, óxido nítrico inalado, ventiladores de alta frequência e oxigenação por membrana extracorpórea tornaram possível salvar mais crianças com SAM. As terapias atuais para SAM são, por natureza, de suporte e não de tratamento da lesão no parênquima pulmonar devido ao mecônio. Uma vez que o líquido mecônio cruza o nível das cordas vocais e atinge o nível do tecido pulmonar, é muito difícil a prevenção da SAM, cabendo a intervenção com terapias de suporte respiratório (BANDIYA P, et al., 2019; CHETTRI S, et al., 2016). Visto isso, a adoção de medidas preventivas possui um valor importante na tentativa de diminuir as mortes causadas pela SAM. Desta forma, percebe-se que o tratamento da enfermidade está relacionado com a disposição de equipamentos para que sua eficácia seja atingida. Por isso, para que haja uma melhora nos índices relacionados a morte por SAM, é necessário que estes equipamentos sejam dispostos no Sistema Único de Saúde, e, para isso, é importante que o Estado brasileiro procure ampliar a rede de saúde e aprimora-la, principalmente em locais de maior demanda, como o estado do Pará.

Além disso, a ampliação da preparação de profissionais para essas intercorrências constitui uma boa ferramenta para evitar óbitos por SAM. Isto se dá devido à importância da aplicação de técnicas afinadas para a realização do tratamento de SAM de maneira assertiva. Desta forma, é importante também a adoção de protocolos obstétricos que tenham como finalidade manter atualizado e assertivo a atuação dos profissionais, a fim de diminuir os riscos de SAM. Outrossim, a adoção de políticas públicas que visem o aumento da qualidade do pré-natal também é uma forma de diminuir as estatísticas sobre a enfermidade (BANDIYA P, et al., 2019; CHETTRI S, et al., 2016).

\section{CONCLUSÃO}

O estado do Pará ocupa o sexto lugar do Brasil em causa de óbitos de recém-nascidos e o primeiro dentro da Região Norte devido a Síndrome de Aspiração Meconial. Observou-se que no estado paraense essa mortalidade esteve presente em $65 \%$ dos casos, com destaque para a capital metropolitana Belém e em algumas cidades com população superior à 100 mil habitantes. Embora os resultados sejam alarmantes, ressalta-se a limitação de sua obtenção, por se tratar apenas da análise de um banco de dados secundários, não tendo sido possível investigar em profundidade as razões de sua elevada ocorrência no estado. Por fim, este estudo fortalece a necessidade de estudos primários sobre os aspectos associados a essa mortalidade, considerando-se os aspectos regionais inerentes às características maternas e neonatais da população paraense, frente às políticas públicas de saúde materno-infantil existentes, a fim de diminuir a ocorrência de óbitos por SAM nos recém-nascidos do estado do Pará. 


\section{REFERÊNCIAS}

1. ALMEIDA MFB, GUINSBURG R. Reanimação do recém-nascido $\geq 34$ semanas em sala de parto. Diretrizes 2016 da Sociedade Brasileira de Pediatria, 26 janeiro de 2016.

2. ANAND V, et al. Activation of toll-like receptors in meconium aspiration syndrome. J Perinatol, 2018;38(2): 127-141.

3. BANDIYA $P$, et al. Surfactant lung lavage vs. standard care in the treatment of meconium aspiration syndrome - a randomized trial. J Trop Pediatr, 2019; 65(2): 114-121.

4. BATRA P, et al. Procalcitonin as Predictor of Bacterial Infection in Meconium Aspiration Syndrome.Am J Perinatol., 2018; 35(8): 769-773.

5. BRASIL. Resolução Normativa № 259, de 17 de junho de 2011. Atendimento dos beneficiários de plano privado de assistência à saúde, Brasília, DF, junho de 2011. Disponível em: < http://www.ans.gov.br/component/legislacao/?view=legislacao\&task=TextoLei\&format=raw\&id=MTc1OA==>. Acesso em: 05 fev. 2020.

6. CHETTRI S, et al. Current Concepts in the Management of Meconium Aspiration Syndrome. The Indian Journal of Pediatrics, 2016; 83(10): 1125-1130.

7. CLEARY GM, WISWELL TE. Meconium-stained amniotic fluid and the meconium aspiration syndrome - an update. Pediatr Clin North Am, 1998; 45: 511-29.

8. DATASUS. Disponível em: http://www2.datasus.gov.br/DATASUS/index.php?area=0205, 1996 a 2016.

9. DARGAVILLE PA. Inflammation in meconium aspiration syndrome-One of many heads of the hydra. Pediatr Pulmonol., 2016;51(6): 555-556.

10. EMPRESA BRASILEIRA DE SERVIÇOS HOSPITALARES (EBSERH); MINISTÉRIO DA EDUCAÇÃO. POP: Fisioterapia na Síndrome da Aspiração de Mecônio. Unidade de Reabilitação do Hospital de Clínicas da Universidade Federal do Triângulo Mineiro. Uberaba: EBSERH - Empresa Brasileira de Serviços Hospitalares, 2015. 16p.

11. FRANÇA EB, et al. Leading causes of child mortality in Brazil, in 1990 and 2015: Estimates from the Global Burden of Disease study. Revista Brasileira de Epidemiologia, 2017; 20(1) 46-60.

12. HAHN S, et al. Lung lavage for meconium aspiration syndrome in newborn infants. Cochrane Database Syst Rev., 2013. 30(4):CD003486.

13. HOFER N, et al. Inflammatory indices in meconium aspiration syndrome. Pediatr Pulmonol., 2016. 51(6):601-606.

14. INSTITUTO BRASILEIRO DE GEOGRAFIA E ESTATÍSTICA. Panorama Estado do Pará. IBGE Cidades. 2019. Disponível em: <https://cidades.ibge.gov.br/brasil/pa/panorama>. Acesso em: 10 mar. 2019.

15. JESUS SP, et al. Neonatologia: síndrome de aspiração de mecônio. CONGREFIP, 2018. Disponível em: <https://www.editorarealize.com.br/revistas/congrefip/trabalhos/TRABALHO_EV069_MD1_SA1_ID134_010420171 81612.pdf>. Acesso em: 10 mar. 2019.

16. LINDENSKOV PHH, et al. Meconium Aspiration Syndrome: Possible Pathophysiological Mechanisms and Future Potential Therapies. Neonatology, 2015; 107(3): 225-230.

17. LISOWSKA-MYJAK B, et al. Correlation between the concentrations of lactoferrin and neutrophil gelatinaseassociated lipocalin in meconium. Biometals, 2018; 31(1): 123-129.

18. LOUIS D, et al. Predictors of mortality in neonates with meconium aspiration syndrome. Indian Pediatr., 2014; 51(8):637-640.

19. MADI JM, et al. Síndrome de aspiração do mecônio: análise de resultados obstétricos e perinatais. RBGO, 2003; 25(2):123-128.

20. NARAYANAN A, et al. PaO2/FiO2 Ratio as Predictor of Mortality in Neonates with Meconium Aspiration Syndrome. Am J Perinatol., 2019; 36(6):609-614.

21. PRATSJAG. Prevention and management of meconium aspiration syndrome. Uptodate. 2018. Disponível em: $<$ https://www.uptodate.com/contents/prevention-and-management-of-meconium-aspiration-syndrome>. Acesso em 15 fev. 2019.

22. SHAIKH M, et al. Detrimental Complications of Meconium Aspiration Syndrome And Their Impact On Outcome. J Ayub Med Coll Abbottabad., 2016; 28(3):506-509.

23. SWARNAM K, et al. Advances in the management of meconium aspiration syndrome. Int $\mathrm{J}$ Pediatr., 2012; 2012(2012):359571. 